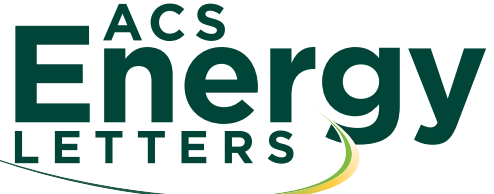

\section{Negative Thermal Quenching in FASnl 3 Perovskite Single Crystals and Thin Films}

\author{
Simon Kahmann, Olga Nazarenko, Shuyan Shao, Oleh Hordiichuk, Mikaël Kepenekian, Jacky Even, \\ Maksym V. Kovalenko, Graeme R. Blake,* and Maria A. Loi*
}

Cite This: ACS Energy Lett. 2020, 5, 2512-2519

ABSTRACT: Formamidinium tin triiodide $\left(\mathrm{FASnI}_{3}\right)$ is a strong contender for sustainable harvesting of solar energy and further optoelectronic applications. So far, only a few studies have considered its fundamental structure-property relationships, given the challenge of ensuring a high material quality. In a concerted effort, we here study high-quality $\mathrm{FASnI}_{3}$ single crystals through a combination of X-ray crystallography, density-functional-theory-based electronic structure calculations, and photoluminescence spectroscopy from room temperature down to $4 \mathrm{~K}$. The luminescence exhibits irregular trends upon cooling with a generally strong intensity increase, but a range of negative thermal quenching, leading to an intensity maximum around $185 \mathrm{~K}$ which is
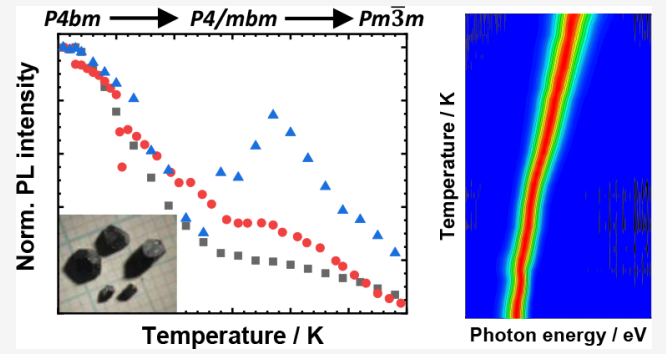
absent in low-quality samples. Differences in the photoluminescence peak position and density-functional-theory-calculated band-gap energies highlight the importance of dynamic processes to the observable properties of FASnI ${ }_{3}$. The presented data offer deeper insight into the temperature-dependent characteristics of this halide perovskite and present opportunities for future exploration of its optoelectronic properties.

$\mathrm{R}$ esearch into metal halide perovskites ( $\mathrm{HaPs}$ ) has initially been driven by the surge in power conversion efficiency (PCE) of photovoltaic cells based on leadcontaining compounds. Nonetheless, it has long spread out into other (opto-)electronic applications, including lightemitting devices, X-ray and photodetectors, and field-effect transistors, accompanied by an ever-growing variety of compositions. Of these compounds, three-dimensional tin iodide-based perovskites are particularly interesting. Not only are they considered to be much more environmentally friendly than lead-containing materials, ${ }^{1}$ but their narrower band gap also allows for a better coverage of the solar spectrum ${ }^{2,3}$ as well as a higher theoretical solar cell PCE than that of their wellperforming relatives based on $\mathrm{Pb}$. An extraordinary long cooling time of hot charge carriers, for $\mathrm{FASnI}_{3}$, in particular ${ }^{4}$ offers an additional interesting opportunity for research into hot carrier solar cells and concentrator devices. ${ }^{5}$

With a near-infrared photoluminescence $(\mathrm{PL})$ that has been reported to be brighter than that of related lead-containing compounds, ${ }^{6}$ FASnI $_{3}$ might furthermore be a promising candidate for applications in optical communication or diagnostics. A large piezoelectric response ${ }^{7}$ as well as the potential for a high figure of merit in thermoelectrics owing to their low thermal conductivity add to the interest in studying the fundamental properties of tin iodide perovskites. ${ }^{8,9}$

The major limiting factor for their successful application is the poor stability toward air exposure, commonly attributed to the easy formation of tin vacancies ${ }^{10,11}$ and the oxidation from $\mathrm{Sn}^{2+}$ into $\mathrm{Sn}^{4+}$. Engineering efforts have led to great advances in the understanding and the mitigation of these processes, ${ }^{12-17}$ as exemplified by recent progress in solar cell performance. $^{18,19}$

With few exceptions, ${ }^{2,20,21}$ research into the optoelectronic properties of tin-based HaPs has so far been focused on thin films. In an effort to broaden these insights and combine different techniques for the analysis of the same samples, we therefore focused our current investigation on single crystals of $\mathrm{FASnI}_{3}$. We employed X-ray diffraction to thoroughly analyze the crystal structure and find three different phases over a temperature range from $298 \mathrm{~K}$ down to $85 \mathrm{~K}$, with results contradicting previous reports. Using these data, we performed density-functional-theory (DFT)-based calculations of the electronic material properties and relate our insights to results obtained from PL spectroscopy on both single crystals and polycrystalline thin films. Our computational findings indicate negligible changes of the band-gap energy for the different crystal structures, which is in accordance with the absence of

Received: May 27, 2020

Accepted: July 7, 2020

Published: July 7, 2020 


\section{Cubic}

(a)

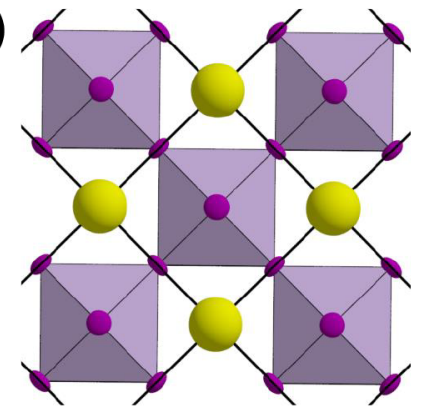

\section{$255 \mathrm{~K}$}

(c)

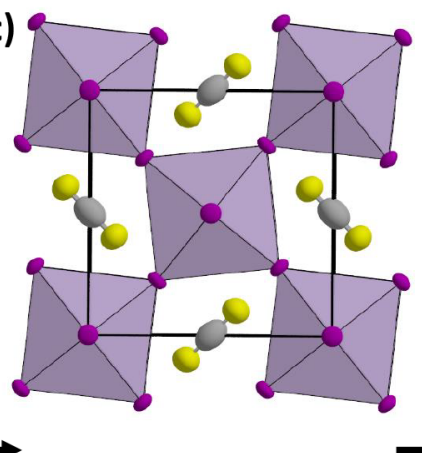

\section{$155 \mathrm{~K}$}

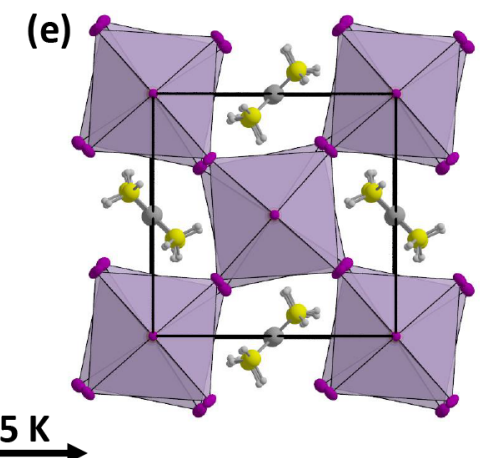

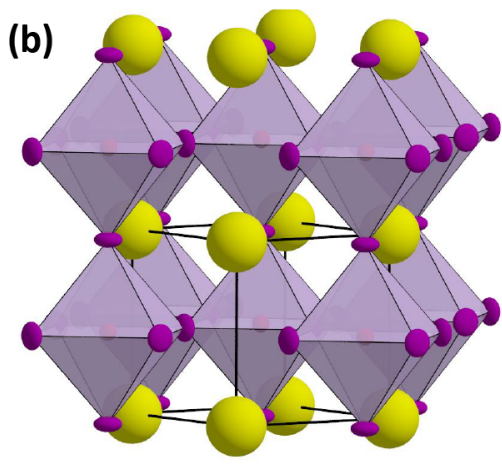

$P m \overline{3} m$

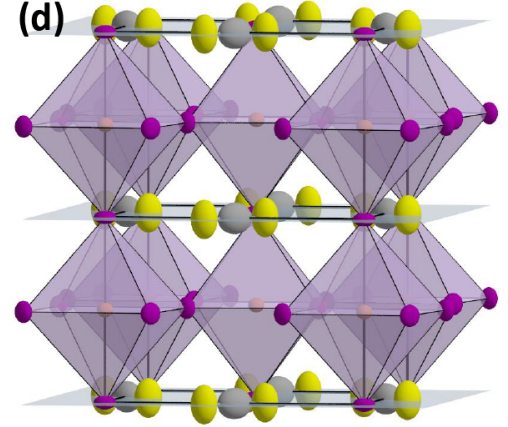

P4/mbm

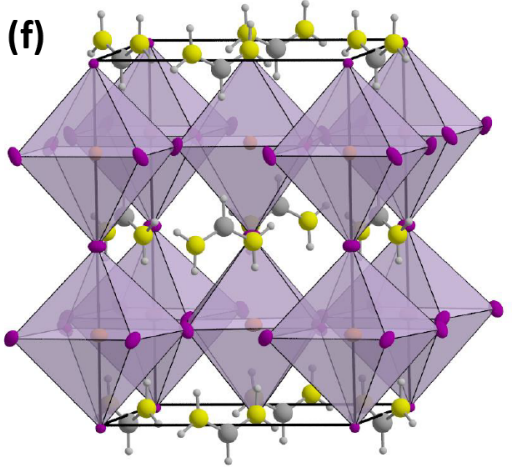

P4bm

Figure 1. $\mathrm{FASnI}_{3}$ undergoes two phase transitions in the temperature range from 300 to $85 \mathrm{~K}$. (a,b) The cubic phase at room temperature contains fully rotationally disordered FA molecules. (c,d) The structure becomes tetragonal at $255 \mathrm{~K}$ due to an in-plane rotation of the SnI 6 octahedra. The orientation of the FA molecules is two-fold disordered due to mirror planes (shaded light blue). (e,f) Doubling of the $c$ lattice parameter occurs at $155 \mathrm{~K}$, as the mirror planes perpendicular to the $c$-axis are removed and the FA molecules become fully ordered. There are now two distinct layers of $\mathrm{SnI}_{6}$ octahedra with different rotation angles.

discontinuities of the PL peak around the phase transitions in single crystals, but highlights the importance of dynamic effects on the band-gap energy through phonons. Inversion symmetry is lost in the low-temperature tetragonal phase, which gives rise to a Rashba-type splitting of both the conduction and valence bands. ${ }^{22}$ The most intriguing result not reported before is a region of negative thermal quenching of the luminescence, which is especially pronounced in high-quality thin films.

Previous work on the temperature-dependent PL of $\mathrm{FASnI}_{3}$ thin films exhibited abrupt changes to the peak luminescence energy, which were tentatively ascribed to changes to the material's crystal structure. ${ }^{6,23}$ We first aim to establish a clear picture of the structural changes that the material undergoes upon cooling from room temperature down to $85 \mathrm{~K}$ (our experimental limit). This will then be used to examine the electronic structure and to compare it with experimental results from PL spectroscopy.

X-ray diffraction was performed on single crystals of $\sim 0.1$ $\mathrm{mm}$ in size (for details of the structure determination and refinement, see the experimental section in the Supporting Information). In line with Mitzi's early assessment, ${ }^{24}$ the material forms a cubic crystal structure (space group $P m \overline{3} m$ ) at room temperature, where the formamidinium (FA) molecules are free to rotate and are completely rotationally disordered. A representation of the structure is given in Figure 1a,b. (See Table 1 for further details.)

Upon cooling, the material undergoes a phase change at 255 $\mathrm{K}$ and becomes tetragonal $(\mathrm{P} 4 / \mathrm{mbm}$, Figure $1 \mathrm{c}, \mathrm{d})$. This is
Table 1. Structural Data Extracted from TemperatureDependent X-ray Diffraction Carried out on Single Crystals (Lattice Parameters Determined at 298, 200, and $100 \mathrm{~K}$ )

\begin{tabular}{|c|c|c|c|c|}
\hline phase & structure & transition & parameters & comment \\
\hline$\alpha$ & cubic & & $a=6.307(2) \AA$ & $\begin{array}{l}\text { FA completely } \\
\text { rotationally } \\
\text { disordered }\end{array}$ \\
\hline$\beta$ & $\begin{array}{l}\operatorname{Pm} \overline{3} m \\
\text { tetragonal }\end{array}$ & $255 \mathrm{~K}$ & $a=b=8.882(1) \AA$ & $\begin{array}{l}\text { in-plane rotation } \\
\text { of } \mathrm{SnI}_{6} \\
\text { octahedra }\end{array}$ \\
\hline & $\mathrm{P} 4 / \mathrm{mbm}$ & & $c=6.270(1) \AA$ & $\begin{array}{l}\text { FA two-fold } \\
\text { disordered by } \\
\text { mirror plane }\end{array}$ \\
\hline$\gamma$ & tetragonal & $155 \mathrm{~K}$ & $a=b=8.838(1) \AA$ & $\begin{array}{l}\text { FA completely } \\
\text { ordered }\end{array}$ \\
\hline & $P 4 b m$ & & $c=12.407(2) \AA$ & \\
\hline
\end{tabular}

chiefly due to an in-plane rotation of the $\mathrm{SnI}_{6}$ octahedra. Simultaneously, the FA molecules become oriented in the $a b$ plane; the space group symmetry imposes linearity on the molecules, which can best be modeled with large ellipsoidal displacement factors in the $c$ direction. This implies a two-fold disordered orientation via the mirror plane that lies perpendicular to $c$. This transition also induces pseudomerohedral twinning, where three domains are formed by successive $120{ }^{\circ} \mathrm{C}$ rotations around the tetragonal [20 $\overline{1}$ ] axis (the reciprocal [10 $\overline{1}]$ axis), as previously observed and discussed for $\mathrm{MAPbI}_{3}{ }^{25} \mathrm{~A}$ further reduction in temperature 
leads to a second phase transition at $155 \mathrm{~K}$, where the $c$-axis doubles due to the complete ordering of FA (Figure 1e,f). The same three twin domains are maintained. The observed reflection conditions are consistent with space group $P 4 / \mathrm{mbm}$ or $P 4 \mathrm{bm}$; structure solution and refinement in the latter gave a significantly better fit $(\mathrm{R} 1=0.0431$ versus 0.0617$)$, implying that the cation ordering breaks inversion symmetry. No further change in structure occurs over the range down to $85 \mathrm{~K}$.

We note that our findings on the structure of $\mathrm{FASnI}_{3}$ conflict with previous studies. Stoumpos et al. studied single crystals and reported changes from orthorhombic (Amm2) at room temperature to a supercell with orthorhombic $I m m 2$ symmetry and four times the volume at $250 \mathrm{~K}$ to a primitive monoclinic structure that could not be solved (close to $100 \mathrm{~K}$ ). ${ }^{2}$ Polycrystalline samples were later studied by Schueller et al., who found the same $P m \overline{3} m$ to $P 4 / m b m$ transition at $250 \mathrm{~K}$ that we observe, but an orthorhombic Pnma structure below 150 $\mathrm{K}^{21}$ Kontos et al. also studied polycrystalline samples and determined a pseudocubic $C 2 \mathrm{~mm}$ structure at room temperature, which transforms into a tetragonal $\mathrm{P} 4 \mathrm{bm}$ phase at $240 \mathrm{~K}$ and then into the same orthorhombic phase as that in ref 21 at $140 \mathrm{~K}^{26}$ This Pnma structure (Pbnm is the setting wherein the pseudotetragonal axes are retained) is inconsistent with our data because we observe many diffraction spots that violate the $h 0 l, h+1=2 n$ reflection condition for an $n$-glide plane. (See the Supporting Information and Figure S2 for further discussion.) We also attempted structure solution and refinement in the orthorhombic space groups Pbam and $P b a 2$, which have the same reflection conditions as $P 4 b m$, but the fits were inferior. (See the Supporting Information.) Because our data do not provide any evidence of inequivalent $a$ and $b$ axes (in terms of lattice parameters, intensities, and reflection conditions in the $h 0 l$ and $0 k l$ reciprocal lattice planes, and the quality of fit when the symmetry is reduced to orthorhombic), we conclude that the tetragonal space group is valid.

On the basis of the previously determined crystal structures, we examined the corresponding electronic band structure (Figure 2) via density functional theory (DFT) following the scheme presented in the computational details section of the Supporting Information (Figures S3 and S4). The band structure for the low-temperature $\mathrm{P} 4 \mathrm{bm}$ phase exhibits a slightly indirect gap due to a Rashba splitting of both the valence and conduction levels at the $\Gamma$-point (Figure 2a). ${ }^{22,27}$ Given their inversion symmetry, the splitting vanishes for the higher temperature phases (Figure $2 \mathrm{~b}, \mathrm{c}$ ). Simultaneously, the band gap moves from the $\Gamma$-point to the $Z$ - and $R$-points in the $P 4 / m b m$ and $P m \overline{3} m$ structures, respectively. As expected for tin-halide perovskites, the valence band maximum mostly presents contributions from the $\mathrm{I}(5 \mathrm{p})$ and $\mathrm{Sn}(5 \mathrm{~s})$ orbitals, whereas the conduction band minimum mostly consists of $\mathrm{Sn}(5 \mathrm{p})$ orbitals (see insets in Figure 2 and projected density of states (DOS) in Figure S5).

Notably, the calculated electronic band-gap energy undergoes a decrease from the low-temperature $\mathrm{P} 4 \mathrm{bm}$ phase to the $P m \overline{3} m$ phase at room temperature (Figure S6a). This result may appear to be inconsistent with the theoretical prediction of a temperature-induced progressive increase in the cell volume and band gap through bond stretching, ${ }^{28}$ as, for example, observed experimentally in the high-temperature phase of $\mathrm{MAPbI}_{3}$ or $\mathrm{FAPbI}_{3},{ }^{25,29}$ but both of these compounds exhibit step-like decreases in the band gap at the lowtemperature structural phase transitions due to the octahedral (a)

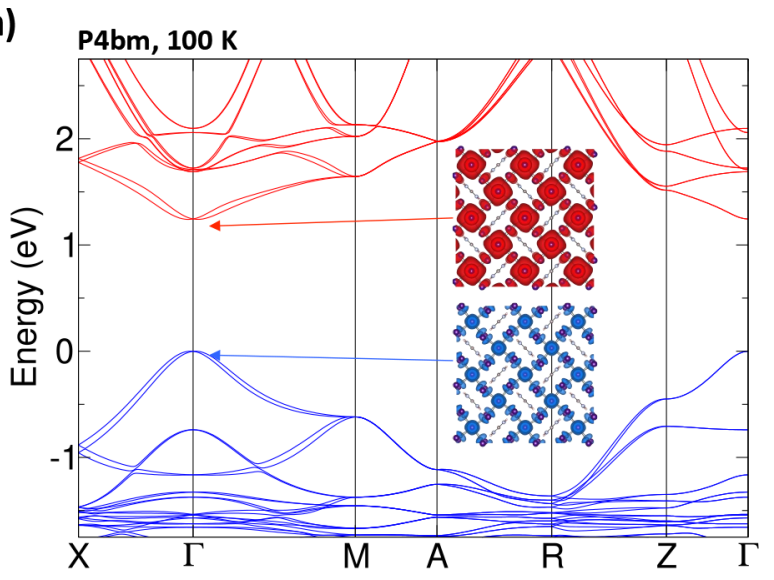

(b)

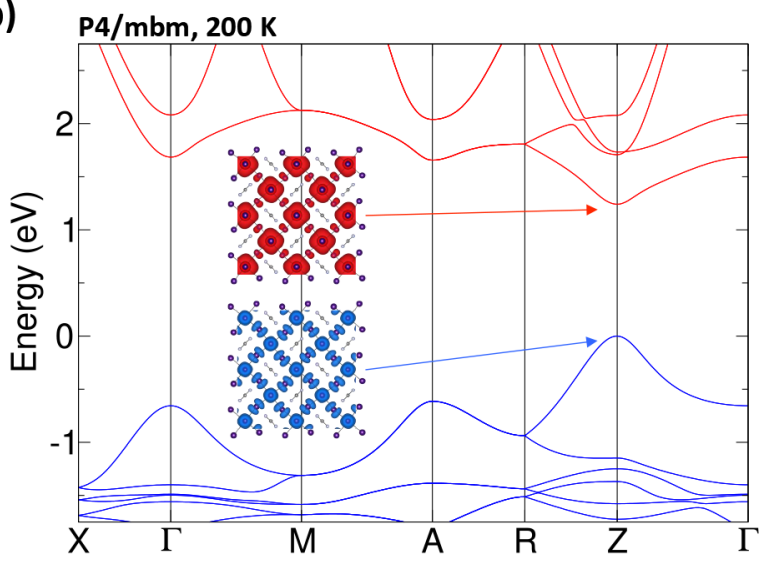

(c)

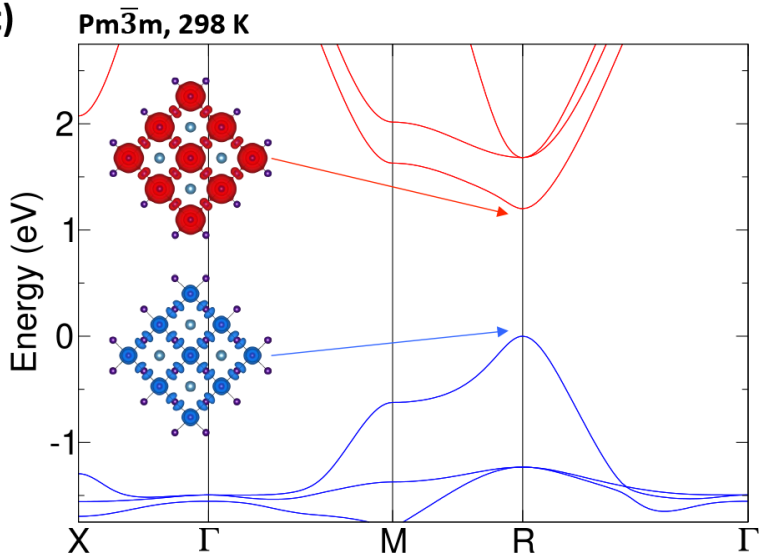

Figure 2. Computed band structures for the (a) $100 \mathrm{~K} \mathrm{P4bm}$, (b) $200 \mathrm{~K} \mathrm{P4} / \mathrm{mbm}$, and (c) $298 \mathrm{~K} \mathrm{Pm} \overline{3} \mathrm{~m}$ structures. Panel c presents a Rashba splitting around the $\Gamma$ point caused by the loss of inversion symmetry and the sizable spin-orbit coupling due to tin and iodide. The insets present the partial charge density computed at the valence band maximum (blue) and conduction band minimum (red).

rotations. In FA-containing lead-based compounds, this effect is already more continuous than in MA-based variants. ${ }^{29}$ Since the experimental optical band gap in $\mathrm{FASnI}_{3}$ undergoes an overall progressive increase upon heating (Figure 3), this seems to be related to the influence of the lattice dynamics on the optical band gap, as discussed later.

From the calculated band structures, we furthermore infer the carrier effective masses, as shown in Figure S6b. Upon increasing the temperature, both the electron and hole effective masses increase (from 0.11 to 0.23 and from 0.06 to 0.13 , 
(a)

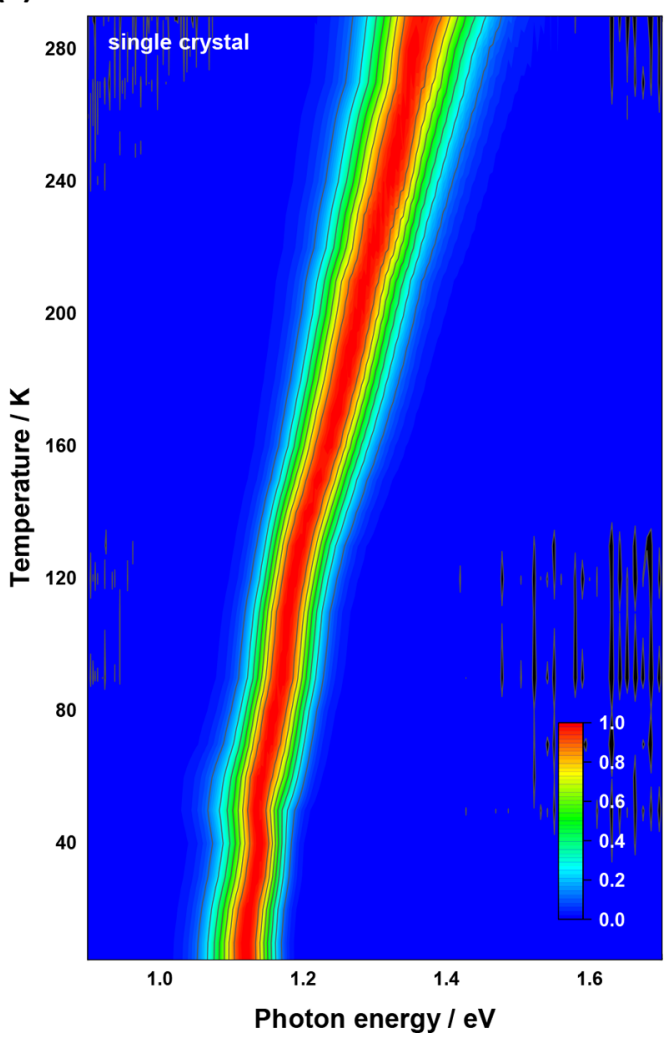

(b)

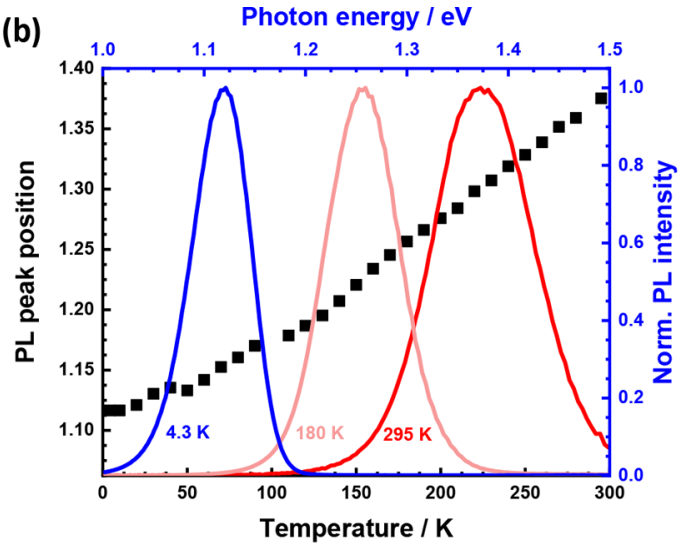

(c)

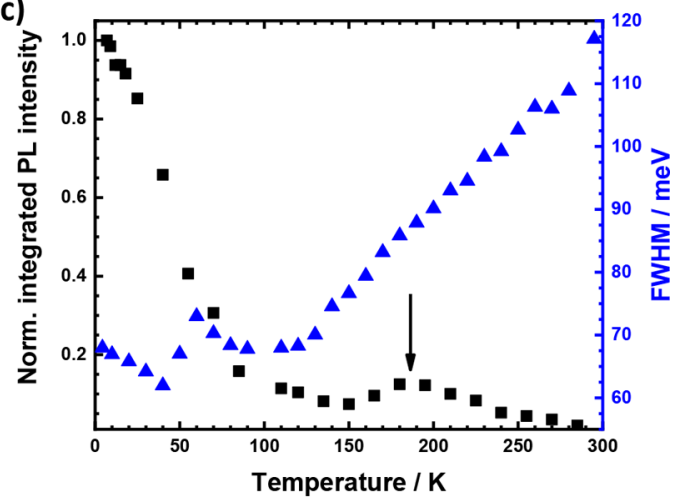

Figure 3. (a) False color plot showing the normalized PL intensity of a single crystal upon cooling to $4.3 \mathrm{~K}$. (b) Peak position and selected spectra. (c) The maximum intensity is reached at cryogenic temperature, but heating reveals a local maximum around $185 \mathrm{~K}$ and increases the line width.

giving rise to reduced effective masses from 0.04 to 0.08 ), with the strongest impact from the $\mathrm{P} 4 \mathrm{bm}$ phase at $100 \mathrm{~K}$ to the $\mathrm{P} 4 /$ $m b m$ phase at $200 \mathrm{~K}$. We note that these reduced masses are about a third of the values obtained from previous work, ${ }^{4}$ which we mainly attribute to the neglect of dynamic effects in current calculations and to the assumption that both carriers contribute to the band-filling effect in ref 4 .

Figure 3 shows the temperature-dependent PL of a $\mathrm{FASnI}_{3}$ single crystal. The false color plot in Figure 3a displays the normalized spectra, indicating the continuous decrease in the peak energy from $\sim 1.37$ to $1.12 \mathrm{eV}$ upon cooling (extracted peak position in Figure $3 \mathrm{~b}$ ). Above $100 \mathrm{~K}$, the shift occurs almost linearly with a gradient of $1.06 \mathrm{meV} / \mathrm{K}$. In contrast to previous observations on thin films (Figure S7) or $\mathrm{FAPbI}_{3}{ }^{29}$ no discontinuity of the peak energy is observed. As previously discussed, our DFT calculations furthermore suggest a small inverse impact of the crystal structure and the thermal expansion on the band-gap energy, $E_{\mathrm{g}}$. Since $E_{\mathrm{g}}$ is generally governed by two aspects, the thermal expansion, $T E$, and electron phonon coupling, $E P, \frac{\mathrm{d} E_{\mathrm{g}}}{\mathrm{d} T}=\left[\frac{\delta E_{\mathrm{g}}}{\delta T}\right]_{T E}+\left[\frac{\delta E_{\mathrm{g}}}{\delta T}\right]_{E P}$, this seems to suggest that the carrier phonon coupling is predominantly responsible for the band-gap change with the temperature increase. Although such assertions have been made before for $\mathrm{Pb}$ compounds, they have recently been contested in a study by Francisco-López et al., ${ }^{30}$ who claim both components to be equally important. The band-gap shift was furthermore attributed to the dynamic off-centering of the $\mathrm{Sn}$ ion by Kontos et al., ${ }^{26}$ the effect of which we cannot capture in our approach.
As illustrated by the exemplary spectra in Figure $3 \mathrm{~b}$ or the blue symbols in Figure 3c, cooling strongly reduces the PL line width (given as fwhm) in the region above $100 \mathrm{~K}$ without any visible impact on the phase transition at either 255 or $155 \mathrm{~K}$. (Also see Figure S8.) The pronounced temperature dependence of the line width above $100 \mathrm{~K}$ indicates the dominant Fröhlich interaction that is generally observed in HaPs. ${ }^{29,31}$ Unfortunately, as we highlight in Figure S9, the broad temperature range $(<100 \mathrm{~K})$, over which the visible line width remains constant, forbids the extraction of meaningful parameters for the coupling constant and effective phonon energy. A virtual independence of the line width at low temperature is often attributed to a large inhomogeneous scattering, $\Gamma_{0}$, for example, due to disorder. Given the reported likelihood of defect formation in tin perovskites, ${ }^{10,11}$ this seems to be a reasonable mechanism. As we shall discuss later, the larger impact of the increasing line width is a consequence of overlapping emitting states at low temperature.

Whereas the previously described results are similar to previous work on $\mathrm{Pb}$-based $\mathrm{HaPs}$, a very irregular trend is displayed by the luminescence intensity. As shown in Figure 3c (black squares), the PL generally brightens upon temperature reduction, but around $185 \mathrm{~K}$, there is a local intensity maximum (black arrow) followed by a region of so-called negative thermal quenching (NTQ), that is, PL reduction upon further cooling. Only at a much lower temperature does the PL intensity strongly increase again. At this local maximum, the intensity reaches close to $20 \%$ of the $4 \mathrm{~K}$ level (peak at $1.25 \mathrm{eV}(993 \mathrm{~nm}$ ) in Figure $3 \mathrm{~b})$. We note that to the best of our knowledge, this behavior has not been observed so 
(a)

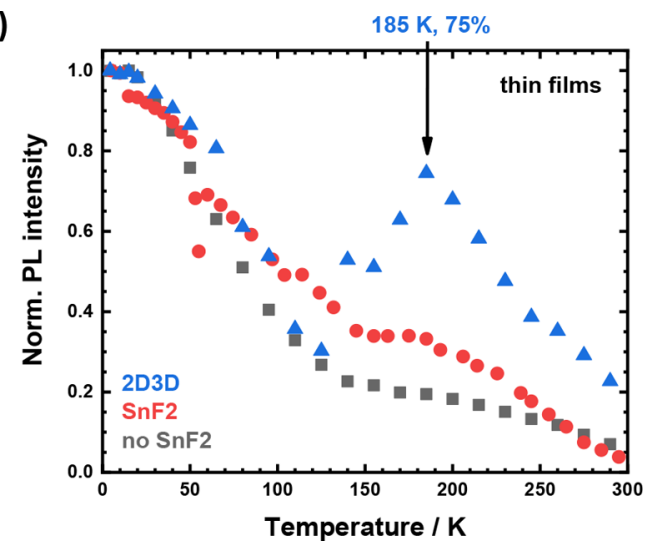

(b)

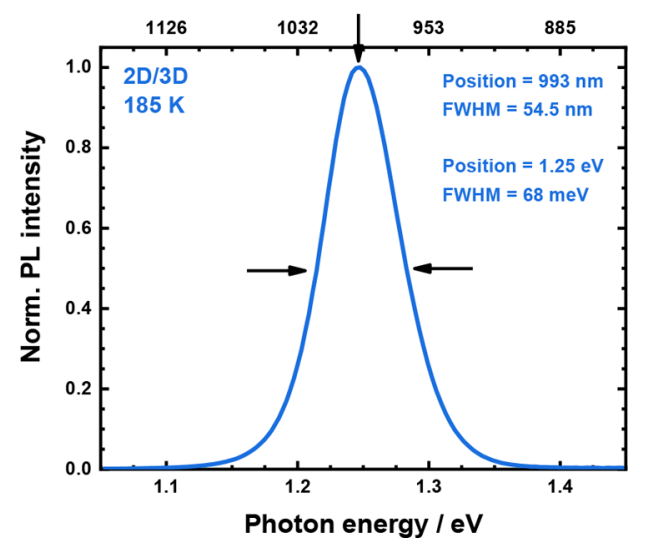

Figure 4. (a) Temperature dependence of $\mathrm{FASnI}_{3}$ thin-film photoluminescence. The 2D/3D film exhibits an extended regime of negative thermal quenching, with a local intensity maximum of $75 \%$ at $185 \mathrm{~K}$. (b) At this temperature, the PL exhibits a single peak at $1.25 \mathrm{eV}(993$ $\mathrm{nm})$ with a width of $68 \mathrm{meV}(54.5 \mathrm{~nm})$.

far. In particular, Kontos et al., who studied the PL intensity of tin-based $\mathrm{HaPs}$ above $100 \mathrm{~K}$, merely observed a continuous PL increase upon cooling. ${ }^{26}$

We thus verified this phenomenon with data on thin films. Following our previous work, ${ }^{14,23,32}$ we examined three kinds of different material quality and found that whereas lower quality films processed without additives or with only $\mathrm{SnF}_{2}$ give rise to a relatively modest intensity increase, high-quality 2D/3D films exhibit even more pronounced NTQ than the single crystals (Figure 4a). The local PL maximum in the 2D/ 3D film at $185 \mathrm{~K}$ reaches $75 \%$ of the value found at $4.3 \mathrm{~K}$. As illustrated in Figure $4 \mathrm{~b}$, the emission at this temperature consists of a single sharp peak at $1.25 \mathrm{eV}(993 \mathrm{~nm})$ in the nearinfrared spectral region with a comparatively narrow fwhm of $68 \mathrm{meV}(54.5 \mathrm{~nm})$. Underlining the impact of the material quality, we note that aged single crystals also do not exhibit any NTQ (Figure S10).

Changes in the crystal structure and thereby in the transition probabilities for radiative recombination $\left(k_{\mathrm{r}}\right)$ can strongly impact the luminescence intensity. An increase in the PL intensity toward a lower temperature is often observed as a consequence of suppressed nonradiative recombination (i.e., a decrease in $k_{\mathrm{nr}}$ ) or the suppressed thermal dissociation of excitons. Strong brightening is thus commonly observed in crystalline materials because phonon-mediated processes freeze out. Systems with disorder or significant shallow trapping, on the contrary, can show reduced luminescence because temperature activation is required for carriers to meet and recombine.

We first consider a possible impact of the radiative recombination rate, as expressed through the bimolecular recombination coefficient, B. A detailed discussion of our approach is given in the computational section of the Supporting Information. In brief, we use the band structures presented in Figure 2 to calculate the Kane parameters for the respective crystal phase and include the effective masses given in Figure $S 6 \mathrm{~b}$ to determine $B$. The resulting values found at 100,200 , and $298 \mathrm{~K}$ amount to $6.19 \times 10^{-10}, 0.92 \times 10^{-10}$, and $0.43 \times 10^{-10} \mathrm{~cm}^{3} \mathrm{~s}^{-1}$, indicating a progressive increase in the recombination coefficient upon cooling (also consider Figure S6c), predominantly driven by the change in effective masses. Although these values somewhat capture the pronounced brightening at low temperature, they also suggest a distinct effect around the $155 \mathrm{~K}$ phase transition, which we do not observe. Moreover, the increase in $B$ contrasts with the NTQ. Both aspects suggest different sources for the intensity dependence.

To analyze the dependence of the PL intensity, it is convenient to separate the behavior into two regions: the drastic increase below roughly $130 \mathrm{~K}$ and the region up to room temperature. As mentioned above and proposed before, ${ }^{23}$ a second emitting state emerges at low temperature, to which we attribute the surge in overall luminescence intensity in that temperature region. Its strong impact can be observed in Figure S11. Although the width of the emission does not allow for clearly distinguishing the features in steady state, we can highlight the impact of the second state using streak camera images, as illustrated in Figure 5.

Extracting the transients, indicated in black in Figure 5a, shows that cooling in this regime increases the overall lifetime of the PL while changing from a virtually monoexponential decay at $130 \mathrm{~K}$ to a biexponential behavior (Figure $5 \mathrm{~b}$ ). We note that the tail exhibits an increasingly long lifetime of close to $8 \mu \mathrm{s}$ at $4 \mathrm{~K}$ (Figure S12). We chose an intermediate time range that allows us to track changes in both the prompt PL and the longer-lived process in Figure $5 \mathrm{~b}$, but it leads to an underestimation of the slow contribution. Simultaneously to the emergence of the long tail, the prompt intensity decreases. (See the inset of Figure 5b.) The extracted parameters of the biexponential fits are given in Table S2 and are depicted in Figure S13. The lifetime of the fast process, $\tau_{\mathrm{f}}$, slightly decreases from approximately 3.5 to $3 \mathrm{~ns}$, whereas the slow process $\tau_{\mathrm{s}}$ exhibits an increase from 12 to $18 \mathrm{~ns}$ (which is grossly underestimated), indicating the importance of the slow process at low temperature.

This trend is furthermore highlighted in Figure 5c, which displays the prompt emission spectra within the first 5 ns and the delayed signal (40-100 ns, see colored regions in a and b; raw data in Figure S14). As suggested by the transients, the prompt intensity slightly decreases upon cooling, and the longlived tail becomes significantly more pronounced. Moreover, whereas the position of both signals generally shifts to longer wavelengths, their separation also increases. (Also consider Figure S15.) Besides the surge in luminescence intensity, this low-energy state thus also explains the previously discussed line-width broadening and the change in the peak energy shift of the steady-state spectra at low temperature shown in Figure $3 \mathrm{~b}$ and $\mathrm{c}$. 

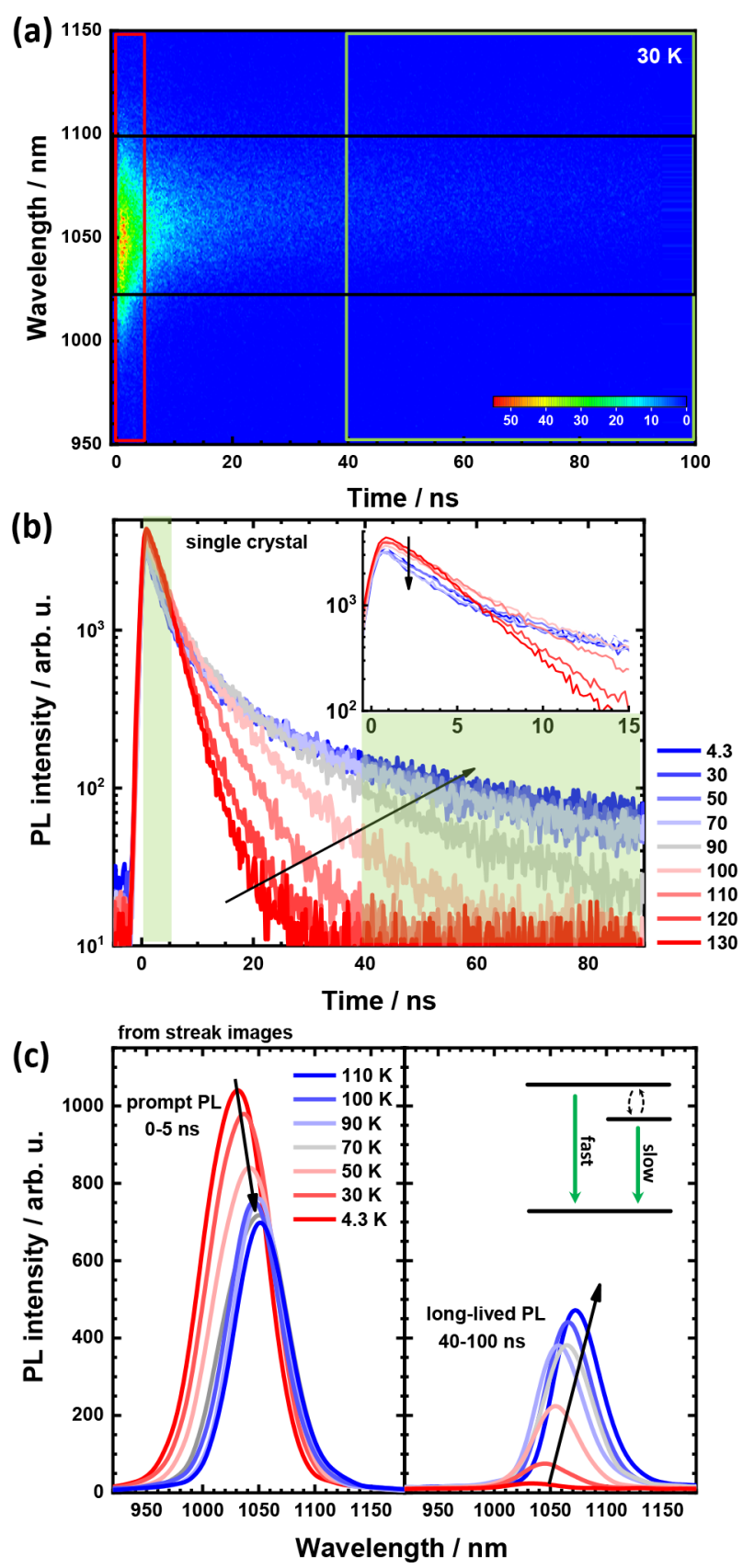

Figure 5. (a) Streak camera image taken at $30 \mathrm{~K}$ to highlight the extraction of the PL transients (black) shown in panel $b$ and the extracted spectra shown in panel c. (b) A monoexponential decay at $130 \mathrm{~K}$ becomes biexponential at lower temperature, where a long-lived tail emerges. The inset depicts the reduction of the PL intensity at early times. (c) Spectra for the prompt (left) and delayed (right) PL illustrate the red-shifted PL and the inverse intensity trend upon temperature reduction.

The detectable onset of the second state just below the phase transition at $155 \mathrm{~K}$ makes the crystal structure a strong contender for the origin of this long-lived process. The slightly indirect character of the band gap due to the Rashba splitting could be responsible for the long lifetime of the lower energy emission. Alternatively, the low-energy transition could be due to shallow trap states (indicated in the inset of Figure 5) or donor-acceptor pairs whose radiative recombination becomes more probable at low temperature. The latter seems especially likely given the increasingly asymmetric shape of the long-lived component at low temperature. Either way, this phenomenon is present in all samples independent of the material quality.

The situation in the temperature region above $\sim 130 \mathrm{~K}$ is more complex. As illustrated by the Arrhenius plots in Figure S10, all samples show an increase in the PL intensity upon initial cooling. In contrast, the NTQ region is clearly linked to the samples' quality, a likely explanation for why it has remained unreported so far. The actual origin of the NTQ remains elusive at this point, but as Figure 4a and Figure S8 show, the important difference is that poor-quality samples exhibit a much more modest PL increase upon cooling from room temperature. Since both single crystals and thin films exhibit the maximum at $185 \mathrm{~K}$ and the NTQ over an extended temperature region, we conclude that first, the cause for the NTQ is intrinsic to $\mathrm{FASnI}_{3}$, but it can easily be obscured by efficient nonradiative processes. Second, the intensity maximum is not a consequence of an abrupt phase transition. An extended region of coexisting crystal phases, as observed for $\mathrm{MAPbI}_{3}$ at low temperature, ${ }^{33}$ also seems unlikely because we do not observe any such evidence in the crystallographic analysis. It could instead be due to the progressive ordering of the FA cations or the progressive rotation of the inorganic cages. It was furthermore proposed that certain types of defects in tin perovskites, such as interstitial $\mathrm{Sn}$ in $\mathrm{MASnI}_{3},{ }^{11}$ exhibit a barrier for carrier trapping. It is possible that such a barrier becomes active upon temperature reduction but is obscured in samples of low quality through additional decay pathways. We expect that deeper insight into this phenomenon will be achieved through a better understanding of defects in $\mathrm{FASnI}_{3}$ as well as from studies of the temperature-dependent phononic properties and a comparison with $\mathrm{MASnI}_{3}$ and $\mathrm{CsSnI}_{3}$.

In conclusion, we used a concerted set of techniques to study the structure-property relationships of formamidinium tin iodide perovskite upon temperature variation. The structure of $\mathrm{FASnI}_{3}$ single crystals was monitored over a range from room temperature down to $85 \mathrm{~K}$, and we found a phase transition from the cubic room-temperature phase to a tetragonal phase around $255 \mathrm{~K}$ and a second tetragonal phase below $155 \mathrm{~K}$ governed by the twisting of the $\mathrm{SnI}_{6}$ cages and the sequential ordering of the FA cations. We used the obtained crystallographic data as a basis for DFT calculations of the band dispersion and studied the behavior close to the fundamental gap. A loss of inversion symmetry due to ordering of the FA cations leads to a Rashba-type splitting of the conduction and valence bands in the low-temperature phase. This might serve to explain the emergence of a second emissive state with a long lifetime observed in temperaturedependent PL spectroscopy, although the large line width of the low-temperature luminescence also suggests a large role of disorder through defects. In stark contrast to the experimental data, we found a small (inverse) impact of the crystal structure on the band-gap energy, adding to the view that dynamic processes are responsible for the temperature dependence of the band gap.

Moderate cooling of $\mathrm{FASnI}_{3}$ gives rise to a strong brightening of the PL in samples of high quality, and a local $\mathrm{PL}$ maximum around $185 \mathrm{~K}$ (at $993 \mathrm{~nm}$ ) forms as a consequence of negative thermal quenching. The origin of the latter phenomenon has not entirely been resolved, but it is clearly linked to the suppression of nonradiative recombination pathways in samples of high quality. The possibility for bright luminescence in the near-infrared spectral region makes 
$\mathrm{FASnI}_{3}$ an even more attractive material for opto-electronic applications that benefit from bright infrared light sources.

\section{ASSOCIATED CONTENT}

\section{SI Supporting Information}

The Supporting Information is available free of charge at https://pubs.acs.org/doi/10.1021/acsenergylett.0c01166.

Experimental details and additional data computational approach and calculations, white-light microscopy image of single crystals, projected density of states and calculated values for band gap and effective masses, and additional spectroscopic results on thin films and single crystals (PDF)

Crystallographic information file of $\mathrm{FASnI}_{3}$ taken at 298 $\mathrm{K}$ (CIF)

Crystallographic information file of $\mathrm{FASnI}_{3}$ taken at 200 $\mathrm{K}$ (CIF)

Crystallographic information file of $\mathrm{FASnI}_{3}$ taken at 100 $\mathrm{K}$ (CIF)

\section{AUTHOR INFORMATION}

\section{Corresponding Authors}

Graeme R. Blake - Solid State Materials for Electronics, Zernike Institute of Advanced Materials, University of Groningen, NL9747 AG Groningen, The Netherlands; 10 orcid.org/00000001-9531-7649; Email: g.r.blake@rug.nl

Maria A. Loi - Photophysics and OptoElectronics Group, Zernike Institute of Advanced Materials, University of Groningen, NL-9747 AG Groningen, The Netherlands; ๑ orcid.org/0000-0002-7985-7431; Email: m.a.loi@rug.nl

\section{Authors}

Simon Kahmann - Photophysics and OptoElectronics Group, Zernike Institute of Advanced Materials, University of Groningen, NL-9747 AG Groningen, The Netherlands; (ㄱ) orcid.org/0000-0001-7784-5333

Olga Nazarenko - Department of Chemistry and Applied Biosciences, ETH Zürich, Zürich CH-8093, Switzerland; EMPA-Swiss Federal Laboratories for Materials Science and Technology, Dübendorf CH-8600, Switzerland

Shuyan Shao - Photophysics and OptoElectronics Group, Zernike Institute of Advanced Materials, University of Groningen, NL-9747 AG Groningen, The Netherlands

Oleh Hordiichuk - Department of Chemistry and Applied Biosciences, ETH Zürich, Zürich CH-8093, Switzerland; EMPA-Swiss Federal Laboratories for Materials Science and Technology, Dübendorf CH-8600, Switzerland; 다이.org/ 0000-0001-7679-4423

Mikaël Kepenekian - Univ Rennes, ENSCR, INSA Rennes, CNRS, ISCR (Institut des Sciences Chimiques de Rennes) UMR 6226, F-35000 Rennes, France; ○ orcid.org/00000001-5192-5896

Jacky Even - Univ Rennes, INSA Rennes, CNRS, Institut FOTON - UMR 6082, F-35000 Rennes, France; (1) orcid.org/ 0000-0002-4607-3390

Maksym V. Kovalenko - Department of Chemistry and Applied Biosciences, ETH Zürich, Zürich CH-8093, Switzerland; EMPA-Swiss Federal Laboratories for Materials Science and Technology, Dübendorf CH-8600, Switzerland; (1) orcid.org/0000-0002-6396-8938

Complete contact information is available at: https://pubs.acs.org/10.1021/acsenergylett.0c01166

\section{Author Contributions}

S.K. and M.A.L. conceived the study. O.N. and O.H. synthesized the single crystals under the supervision of M.V.K. S.S. fabricated thin-film samples. G.R.B. performed and analyzed the X-ray crystallographic investigation. M.K. and J.E. performed and analyzed the computational analysis. S.K. performed and analyzed the optical spectroscopy experiments and wrote the first draft. All authors subsequently improved upon it.

\section{Notes}

The authors declare no competing financial interest.

The CIF data for the 200 and $100 \mathrm{~K}$ structure have been deposited in the Cambridge Crystallographic Data Centre (CCDC) under deposition numbers 2004417 and 2004418, respectively

\section{ACKNOWLEDGMENTS}

Arjen Kamp and Teo Zaharia are kindly thanked for technical support. S.K. acknowledges a research fellowship (grant no. 408012143) awarded by the Deutsche Forschungsgemeinschaft (DFG). This work was partially financed through the Materials for Sustainability (Mat4Sus) programme (739.017.005) of The Netherlands Organisation for Scientific Research (NWO). J.E. acknowledges Institut Universitaire de France for funding. This project has received funding from the European Union's Horizon 2020 research and innovation Programme under the grant agreement no. 862656. The information and views set out in the abstracts and presentations are those of the authors and do not necessarily reflect the official opinion of the European Union. Neither the European Union institutions and bodies nor any person acting on their behalf may be held responsible for the use which may be made of the information contained herein. This work was granted access to the HPC resources of [TGCC/CINES/ IDRIS] under the allocation 2019-A0070907682 made by GENCI. M.K. and J.E. thank Boubacar Traoré for providing atomic pseudopotentials as well as Laurent Pedesseau and Shuxia Tao for fruitful discussions about the DFT-1/2 approach. We thank Dr. Costas Stoumpos for kindly providing the low-temperature crystal structure reported in ref 26 .

\section{REFERENCES}

(1) Li, J.; Cao, H.-L.; Jiao, W.-B.; Wang, Q.; Wei, M.; Cantone, I.; Lï, J.; Abate, A. Biological impact of lead from halide perovskites reveals the risk of introducing a safe threshold. Nat. Commun. 2020, 11,310 .

(2) Stoumpos, C. C.; Malliakas, C. D.; Kanatzidis, M. G. Organic Tin and Lead Iodide Perovskites with Organic Cations: Unique Semiconductors, with Phase Transitions and Near-infrared Photoluminescent Properties. Inorg. Chem. 2013, 52, 9019-9038.

(3) Tao, S.; Schmidt, I.; Brocks, G.; Jiang, J.; Tranca, I.; Meerholz, K.; Olthof, S. Absolute energy level positions in tin- and lead-based halide perovskites. Nat. Commun. 2019, 10, 2560.

(4) Fang, H.-H.; Adjokatse, S.; Shao, S.; Even, J.; Loi, M. A. Longlived hot-carrier light emission and large blue shift in formamidinium tin triiodide perovskites. Nat. Commun. 2018, 9, 243.

(5) Kahmann, S.; Loi, M. A. Hot carrier solar cells and the potential of perovskites for breaking the Shockley-Queisser limit. J. Mater. Chem. C 2019, 7, 2471-2486.

(6) Parrott, E. S.; Green, T.; Milot, R. L.; Johnston, M. B.; Snaith, H. J.; Herz, L. M. Interplay of Structural and Optoelectronic Properties in Formamidinium Mixed Tin-Lead Triiodide Perovskites. Adv. Funct. Mater. 2018, 28, 1802803. 
(7) Pandey, R.; SB, G.; Grover, S.; Singh, S. K.; Kadam, A.; Ogale, S.; Waghmare, U. V.; Rao, V. R.; Kabra, D. Microscopic Origin of Piezoelectricity in Lead-Free Halide Perovskite: Application in Nanogenerator Design. ACS Energy Lett. 2019, 4, 1004-1011.

(8) Mettan, X.; Pisoni, R.; Matus, P.; Pisoni, A.; Jaćimović, J.; Náfrádi, B.; Spina, M.; Pavuna, D.; Forró, L.; Horváth, E. Tuning of the Thermoelectric Figure of Merit of $\mathrm{CH}_{3} \mathrm{NH}_{3} \mathrm{MI}_{3}(\mathrm{M}=\mathrm{Pb}, \mathrm{Sn})$ Photovoltaic Perovskites. J. Phys. Chem. C 2015, 119, 11506-11510.

(9) Liu, T.; Zhao, X.; Li, J.; Liu, Z.; Liscio, F.; Milita, S.; Schroeder, B. C.; Fenwick, O. Enhanced control of self-doping in halide perovskites for improved thermoelectric performance. Nat. Commun. 2019, 10, 5750.

(10) Shi, T.; Zhang, H.-S.; Meng, W.; Teng, Q.; Liu, M.; Yang, X.; Yan, Y.; Yip, H.-L.; Zhao, Y.-J. Effects of organic cations on the defect physics of tin halide perovskites. J. Mater. Chem. A 2017, 5, 1512415129.

(11) Meggiolaro, D.; Ricciarelli, D.; Alasmari, A. A.; Alasmary, F. A. S.; De Angelis, F. Tin versus Lead Redox Chemistry Modulates Charge Trapping and Self-Doping in Tin/Lead Iodide Perovskites. J. Phys. Chem. Lett. 2020, 11, 3546-3556.

(12) Leijtens, T.; Prasanna, R.; Gold-Parker, A.; Toney, M. F.; McGehee, M. D. Mechanism of Tin Oxidation and Stabilization by Lead Substitution in Tin Halide Perovskites. ACS Energy Lett. 2017, 2, 2159-2165.

(13) Ke, W.; Stoumpos, C. C.; Spanopoulos, I.; Mao, L.; Chen, M.; Wasielewski, M. R.; Kanatzidis, M. G. Efficient Lead-Free Solar Cells Based on Hollow \{en\}MASnI 3 Perovskites. J. Am. Chem. Soc. 2017, 139, 14800-14806.

(14) Shao, S.; Liu, J.; Portale, G.; Fang, H.-H.; Blake, G. R.; ten Brink, G. H.; Koster, L. J. A.; Loi, M. A. Highly Reproducible SnBased Hybrid Perovskite Solar Cells with 9\% Efficiency. Adv. Energy Mater. 2018, 8, 1702019.

(15) Adjokatse, S.; Kahmann, S.; Duim, H.; Loi, M. A. Effects of strontium doping on the morphological, structural, and photophysical properties of $\mathrm{FASnI}_{3}$ perovskite thin films. APL Mater. 2019, 7, 031116.

(16) Shao, S.; Dong, J.; Duim, H.; ten Brink, G. H.; Blake, G. R.; Portale, G.; Loi, M. A. Enhancing the crystallinity and perfecting the orientation of formamidinium tin iodide for highly efficient Sn-based perovskite solar cells. Nano Energy 2019, 60, 810-816.

(17) Chen, M.; Ju, M.-G.; Garces, H. F.; Carl, A. D.; Ono, L. K.; Hawash, Z.; Zhang, Y.; Shen, T.; Qi, Y.; Grimm, R. L.; Pacifici, D.; Zeng, X. C.; Zhou, Y.; Padture, N. P. Highly stable and efficient allinorganic lead-free perovskite solar cells with native-oxide passivation. Nat. Commun. 2019, 10, 16.

(18) Konstantakou, M.; Stergiopoulos, T.; et al. A critical review on tin halide perovskite solar cells. J. Mater. Chem. A 2017, 5, 1151811549.

(19) Ke, W.; Stoumpos, C. C.; Kanatzidis, M. G. Unleaded" Perovskites: Status Quo and Future Prospects of Tin-Based Perovskite Solar Cells. Adv. Mater. 2019, 31, 1803230.

(20) Dang, Y.; Zhou, Y.; Liu, X.; Ju, D.; Xia, S.; Xia, H.; Tao, X. Formation of Hybrid Perovskite Tin Iodide Single Crystals by TopSeeded Solution Growth. Angew. Chem., Int. Ed. 2016, 55, 34473450.

(21) Schueller, E. C.; Laurita, G.; Fabini, D. H.; Stoumpos, C. C.; Kanatzidis, M. G.; Seshadri, R. Crystal Structure Evolution and Notable Thermal Expansion in Hybrid Perovskites Formamidinium Tin Iodide and Formamidinium Lead Bromide. Inorg. Chem. 2018, 57, 695-701.

(22) Stroppa, A.; Di Sante, D.; Barone, P.; Bokdam, M.; Kresse, G.; Franchini, C.; Whangbo, M.-H.; Picozzi, S. Tunable ferroelectric polarization and its interplay with spin-orbit coupling in tin iodide perovskites. Nat. Commun. 2014, 5, 5900.

(23) Kahmann, S.; Shao, S.; Loi, M. A. Cooling, Scattering, and RecombinationâÁThe Role of the Material Quality for the Physics of Tin Halide Perovskites. Adv. Funct. Mater. 2019, 29, 1902963.
(24) Mitzi, D.; Liang, K. Synthesis, Resistivity, and Thermal Properties of the Cubic Perovskite $\mathrm{NH}_{2} \mathrm{CH}=\mathrm{NH}_{2} \mathrm{SnI}_{3}$ and Related Systems. J. Solid State Chem. 1997, 134, 376-381.

(25) Fang, H.-H.; Raissa, R.; Abdu-Aguye, M.; Adjokatse, S.; Blake, G. R.; Even, J.; Loi, M. A. Photophysics of Organic-Inorganic Hybrid Lead Iodide Perovskite Single Crystals. Adv. Funct. Mater. 2015, 25, 2378-2385.

(26) Kontos, A. G.; Kaltzoglou, A.; Arfanis, M. K.; McCall, K. M.; Stoumpos, C. C.; Wessels, B. W.; Falaras, P.; Kanatzidis, M. G. Dynamic Disorder, Band Gap Widening, and Persistent Near-IR Photoluminescence up to At Least $523 \mathrm{~K}$ in $\mathrm{ASnI}_{3}$ Perovskites (A = $\mathrm{Cs}^{+}, \mathrm{CH}_{3} \mathrm{NH}_{3}{ }^{+}$and $\left.\mathrm{NH} 2 \_\mathrm{CH}=\right) \mathrm{NH}_{2}{ }^{+}$. J. Phys. Chem. C 2018, 122, 26353-26361.

(27) Kepenekian, M.; Robles, R.; Katan, C.; Sapori, D.; Pedesseau, L.; Even, J. Rashba and Dresselhaus Effects in Hybrid OrganicInorganic Perovskites: From Basics to Devices. ACS Nano 2015, 9, 11557-11567.

(28) Boyer-Richard, S.; Katan, C.; Traoré, B.; Scholz, R.; Jancu, J.M.; Even, J. Symmetry-Based Tight Binding Modeling of Halide Perovskite Semiconductors. J. Phys. Chem. Lett. 2016, 7, 3833-3840.

(29) Fang, H.-H.; Wang, F.; Adjokatse, S.; Zhao, N.; Even, J.; Antonietta Loi, M. Photoexcitation dynamics in solution-processed formamidinium lead iodide perovskite thin films for solar cell applications. Light: Sci. Appl. 2016, 5, No. e16056.

(30) Francisco-López, A.; Charles, B.; Weber, O. J.; Alonso, M. I.; Garriga, M.; Campoy-Quiles, M.; Weller, M. T.; Goñi, A. R. Equal Footing of Thermal Expansion and Electron-Phonon Interaction in the Temperature Dependence of Lead Halide Perovskite Band Gaps. J. Phys. Chem. Lett. 2019, 10, 2971-2977.

(31) Wright, A. D.; Verdi, C.; Milot, R. L.; Eperon, G. E.; PérezOsorio, M. A.; Snaith, H. J.; Giustino, F.; Johnston, M. B.; Herz, L. M. Electron-phonon coupling in hybrid lead halide perovskites. Nat. Commun. 2016, 7, 11755.

(32) Dong, J.; Shao, S.; Kahmann, S.; Rommens, A. J.; HermidaMerino, D.; ten Brink, G. H.; Loi, M. A.; Portale, G. Mechanism of Crystal Formation in Ruddlesden-Popper Sn-Based Perovskites. Adv. Funct. Mater. 2020, 30, 2001294.

(33) Dobrovolsky, A.; Merdasa, A.; Unger, E. L.; Yartsev, A.; Scheblykin, I. G. Defect-induced local variation of crystal phase transition temperature in metal-halide perovskites. Nat. Commun. 2017, 8, 34 . 